Aus der I. chirurgischen Abteilung des Rudolf-Virchow-Krankenhauses, Berlin. (Direktor: Prof. Dr. M ühs a m.)

\title{
Ersatz der Tibia durch eine Magnalium-Prothese.
}

\author{
Von Oberarzt Dr, Gerhard Golm, Assistent. der Khnlk.
}

Mit 5 Abbildungen.

Während die Geschichte der plastischen (Operationen, soweit sie sich auf die Weichteile erstrecken, weit zurückreicht - übten doch die Inder schon eine Rhinoplastik -, fallen die Anfänge der plastischen Knochenchirurgie in die I. Hälfte des vorigen Jahrhunderts. Die Versuche, größere Knochendefekte zu ersetzen, gehen in erster Linie auf $\mathrm{Juli}$ us Wolff und Ollier zurück. Sie erkannten zuerst die knochenneubildende Eigenschaft des Periosts und Knochenmarks, ihre Experimente über Implantation, Regeneration ron Knochen legten den Grund zur heutigen Osteoplastik. Aber ihre an Tieren ganz erfolgreichen Experimente konnten, auf den Menschen übertragen, noch keinen wesentlichen Fortschritt bedeuten. Die Asepsis steckte noch in den Kinderschuhen, die Wunden wollten nicht heilen. Von einer freien Knochentransplantation wollte man gar nichts wissen, man scheute sich, eine neuc Wunde zu setzen. Auf der Suche nach einem knochenähnlichen Material fand man das Elfenbein am seeignetsten. Schon Dieffenbach benutzte im Jahre $184^{6}$ in der Knochenchirurgie Elfenbein, das er in nicht heilenwollende Knochenfragmente eintrieb, um die Knochenneubildung anzufachen. Dieses Material griff man in den go er Jahren wieder auf, um großere Knochendefekte zu decken, und so gelang es insbesondere Gluck als erstem mehrfach, auch großere Knochendefekte durch Elfenbein zu ersetzen. So ersetzte er im Jahre I 898 das untere Drittel einer resezierten Ulna durch Elfenbein. Das Elfenbein heilte vollkommen ein, ja der Patient zog sich ${ }^{1}:$ Jahr spater eine Fraktur am Implantationsorte zu, welche mit knocher- 
nem (alluw weder zusammenheilte. Das Elfenbeinstück war durch Substitutionsostose, wie Gluck diesen Vurgang nannte, rollkommen ersetzt worden. Es gelang auch (i luck, toten Knochen zur Einheilung zu bringen. Er benutzte dazu Knochen seines Pariser Skeletts aus dem Jahre I $8,3,3$ und er konnte stolz auf den Erfolg sem, als er hörte, daß dieser Patient als Landsturmmann den Krieg I01 4--I\& gegen Frankreich mitgemacht hat. Giordano berichtel ebenfalls gute Erfolge mit Elfenbeinimplantationen. Er setzte in emen Tibiadefekt em $23 \mathrm{~cm}$ langes und $18-22 \mathrm{~mm}$ breites Fisenstuck, welches am oberen und mteren Ende einen Meniscus aus lilfenbein trug. Wohl bahubrechend auf diesem iebiete der Aloplastik i'latsk mit körpertremdem Material) war aber doch (i) u k mit semer Invaginationsmethode. Er stelle I So5 auf dem Chrurgenkongrel3 eine Anzalil ron Versuchstieren vor, unter anderm zeigte er Hunde, denen er ganze Metallapparate implantiert hatte. Aber die Erfolge waren beim Menschen mit diesen komplizierten Apparaten keine dauernden. Die Prothesen mulbten in den meisten Fallen wegen Eiterung wieder entfernt werden. Aher eins war dann doch stets erreicht worden: en hatte sich entlang dieser Fremdkorpersihiene, die gewissermaben als Leitband diente, Knochen neu sebıldet. Auf der Suche nach einem Material, das zwar eine Stutze biete, aber allmählich im Kirper resorbiert würde, kam Ciluck aut den Gedanken, als Knochencrsatz ein metallisches Präparat ,Magnalium“ zu verwenden. Magnalium ist ein Gemisch von Magnesium und Aluminium und wurde cuerst ron Payr im Jahre I yoo als resorbierbare Prothese zur Vereinigung getrennter Gefäßlumina herausgegeben. Die I. Versuche l'ays gehen in das Jahr 1892 zurück. Er konnte feststellen, daß sich das Magnalium, in dünnen Stucken in menschliches Gewebe gebracht, leicht lust. Iuf die chemischen Vorgange kann ich leider hier nicht eingehen, da es zu weit führen wurde. Ich verweise hier auf die aus. führlichen Angaben Payrs (Verhandl. d. deutsch. Gesellschaft f. Chir. 1900, XXXV). Dieses Magnalium war dank seiner chemischen Zusammensctzung gut zu rerarbeiten. In der Hoffnung, nun eine brauchbare resorbicrbare Prothese gefunden zu haben, ging Gluck an dic Implantation von Nagnalium-Stäben. Während die Gefaßprothesen Pay s restlos resorbiert wurden, ist dies bei den Magnalium-Prothesen $\mathrm{G} l \mathrm{ucks}$ in Extremitäten nicht beobachtet 
worden. Freilich konnte Gluck einen Magnalium-Stab zeigen, den er bei Osteomyelitis des Femur nach Resektion ron $10 \mathrm{~cm}$ Knocheri implantiert hatte. Er blicb $5^{-1}, 2$ Monate liegen, mußte dann aber entfernt werden, da sich eine Fistel gebildet hatte, die nicht heilen wollte. An diesem Stabe licßen sich Rinnen, Furchen und Locher erkennen, die das angrenzende Gewebe hineingefressen hattc. Wenn auch der Stab nicht resorbert worden war und darum entfernt werden mußte, eins hatte er lorh erreicht, die Knochenneubildung war machtig angeregt worden, so dal3 nach seiner Entfernung die Extremitat schnell gebrauchsfahig wurde. Warum die Resorption nicht eine vollkommene war, ist nicht näher untersucht worden. Wahrscheinlich liegt es doch wohl nur an der weit gröberen Masse von Material, das bei der Knochen. ersatzmethode verwendet werden mußte, als bei den dünnen, feinen Magnalium-Prothesen, welche die Kontinutät ron Gefaßen wieder herstellen sollten.

Fragen wir uns nun, was aus solcher Mctallprothese wird, wenn sie im Korper nicht resorbitit wird, nicht ausgestoßen und auch nicht operativ wieder entfernt wird! Kann sie nicht unter Umstanden zu schweren Funktionsstơrungen und Wachstumsbeschränkungen fuhren, zumal dann, wenn es sich um ein jugendlichen Individuum handelt? Diese Frage ist darum so aktucll, weil gerade einige Chirurgen, insbesondere Lange, fur Fremdkörperimplantationen im allgememen wieder eintreten (Zelluloid-, Horn-, Eltenbemstifte). Wie rorsichtig man aber bel jugendlichen Individuen, besonders bei zweiknochigen Extremitäten, mit der Fremdkörperimplantation sein soll, beweist folgender Patient, den wir zu beobachten Gelegonheit hatten.

Im April rozo fand ein Pat bei unn Aufnahtme, der kur folgenden Befund aufwies: Junger Mann, dessen rechter Iintersohenkel um g) cm verkur th ist. An der Strecksente des Unterschenkels innen eine $2 z \mathrm{~cm}$ lange Narbe An der (rrenze zwischen mittlerem und unterem Drittel innen 2 Ulcera mit Fistên. Drc Sonde stößt hier aul Rauhigkciten. Der Fuß stcht in Klumpfußstellung, dic Fibula int in ihrem untcren Drittel stark rcrkrimmt, nach innen gebogen. Starke Atrophie cler Wadenmuskulatur, der rechte Fuß ist bedeutend kleiner als dcr linke Pat. traggt einen Sticfel mit crhohtem Absat Der Gangr ist hinkend und scheint dem Pat Schmerzen zu boreiten.

Fig I laßt dor Verhalnisse ancrhaulich erkennen Die Fisteln 
an der Innenseite des Unterschenkels im unteren Drittel sind deutlich sichtbar, ebenso die Klumpfußstellung und dic Verbiegung der Fibula.

Wir ließen eine Róntgenaufnahme anfertgren und sahen an stelle der Tibia Diaphyse einen Fremdkörper aus Metall. Im Jahre igos war dem Pat. im Kinderkrankenhatus Berlın ron Gluck dieser Fremdkörper implantiert worden. Der liebenswurdigen Auskunft des Herrn Prof. Dr. Th. Gluck verdanke ich folgende Angaben: „Es handelte sich damals bei dem Pat. im Jahre 1908 um eine flaschenförmige Knochen-Tuberkulose der ganzen Tibia-Diaphyse, aus-

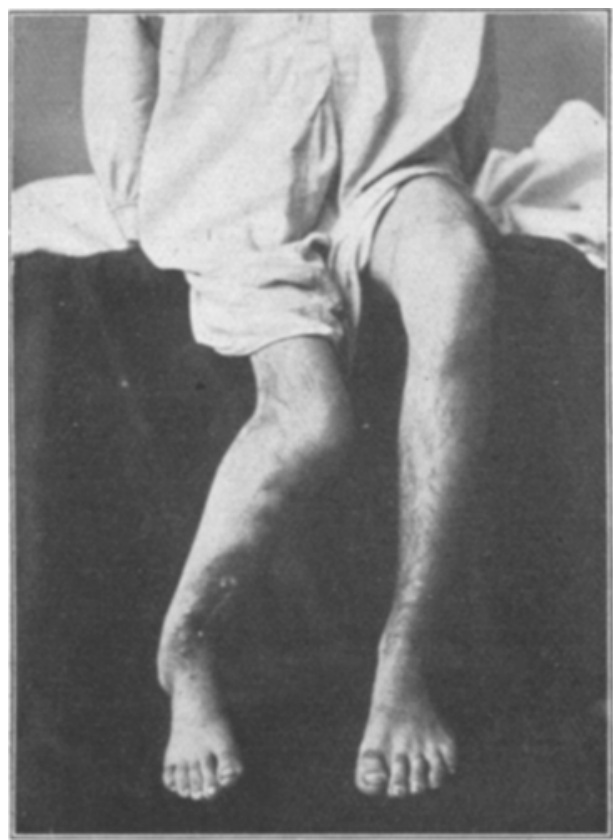

Fig. I. gudehnte käsige Sequester, Ostermyelitis tubercu. losa, schwere Weichteil. 'Tuberkulose mit Fistelbil. dung Die sanze Trbia. Diaphyse wurde reseziert, das Periost muBte ebentalls zum größten Teil mit entfernt werden Die restierenden Gelenkenden der Tibia wurden durch ene eingepflanzte MagnaliumProthese fest mi einander verbunden, die Haut daruber vernaht Die fistel. lose Einheilung konnte bis zum Jahre I9I5 verfolgt werden, also 7 Jahre post operationem liat. trug emen Hessingschen Apparat und ging trotz Verkurzung ganz gut."

Der Pat stellte sich bei Gluck nicht wieder vor und kam, wie oben gesagt, I920 in unsere Bchandlung. Dic Rontgcnaufnahme, Fis. 2 und 3, zeigt uns folgendes: Die ganze Tibia-Draphyse ist durch einen nach distal zu spitzer werdenden, metallischen Stab erietzt, dessen Konturen glatt sind Keine Furchen und Löcher als Zeichen von Resorptionsvorgängen. Um die Implantationsstelle an der prox. Epiphyse Knochenneubildung in Spangenform. Eigenartiges Wachs. tum der Fibula, Verbiegung im distalen Drittel nach innen. Fehlerhafte Stellung der distalen Gelenkflache der Tibı, die schrag nicht horizontal vorlauft. Auffallende Osteoporose der Fußwurzelknochen. 
Von dem einzuschlagenden Heilverfahren kim wegen der hochgradigen Verkursung und Deformirrung des Unterschenkels, der schweren Weichtcil-Tuberkulose mit Fistelbildung und bei dem Fehlen

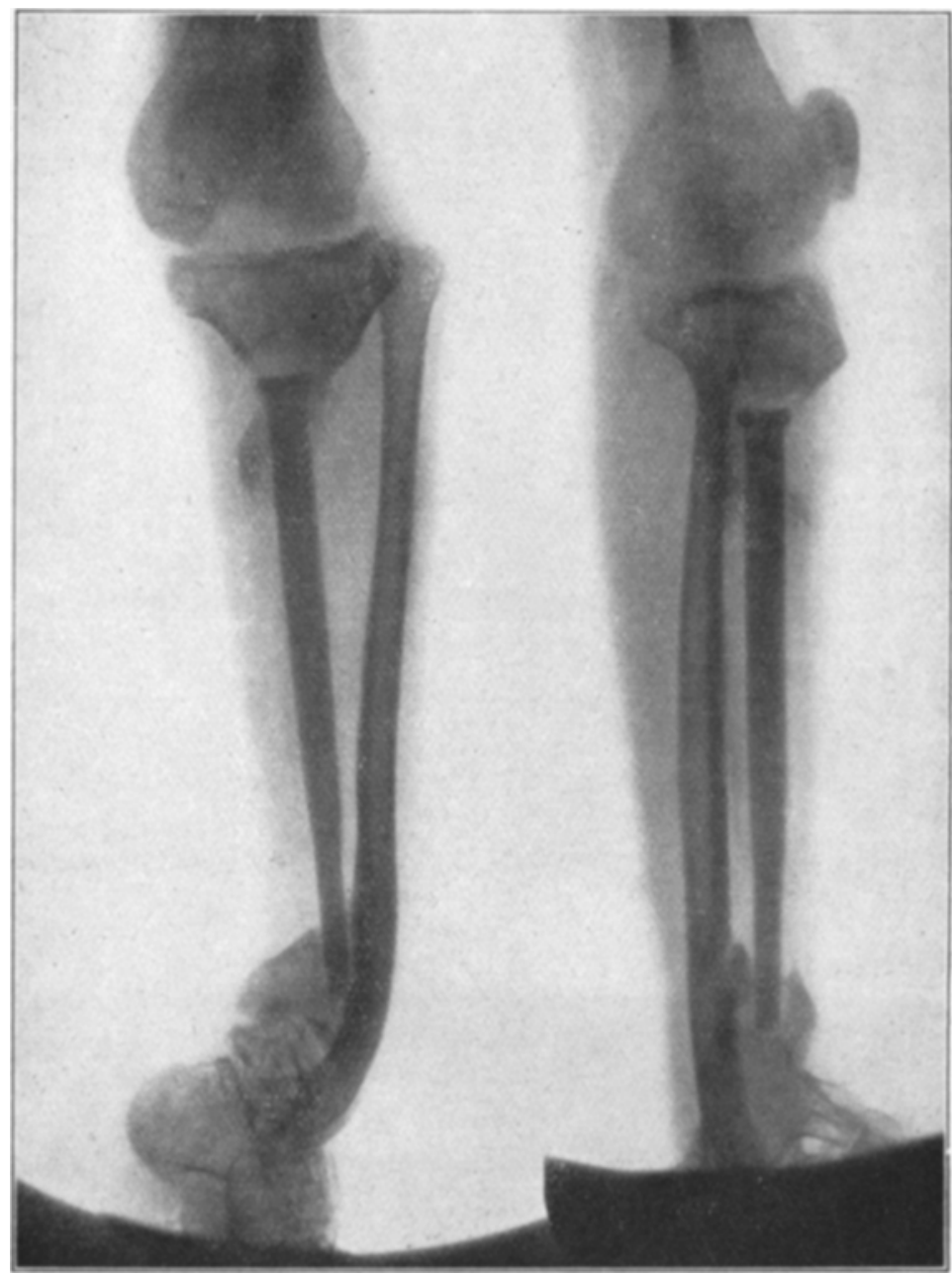

Fig. 2 und 3 .

der Tibia Dialthyse nur eine Amputation in Fratre Dienelbe wurde am I4. IV. I920 tran kondylär vorgenommen.

Das durch die Amputation gewonnene Präparat zeigt uns Fig. 4 und 5. Hier sehen wir wieder deutlich am Magnalıum-Stabe keinerlei 
Teränderungen, die auf eine Resnrprion des Materials schlicßen lassen. Weiter zeigt uns das Praparat noch besser als das Rontgenbild dic eigenartige Verkrummung der Fibula in distalen Drittel und die
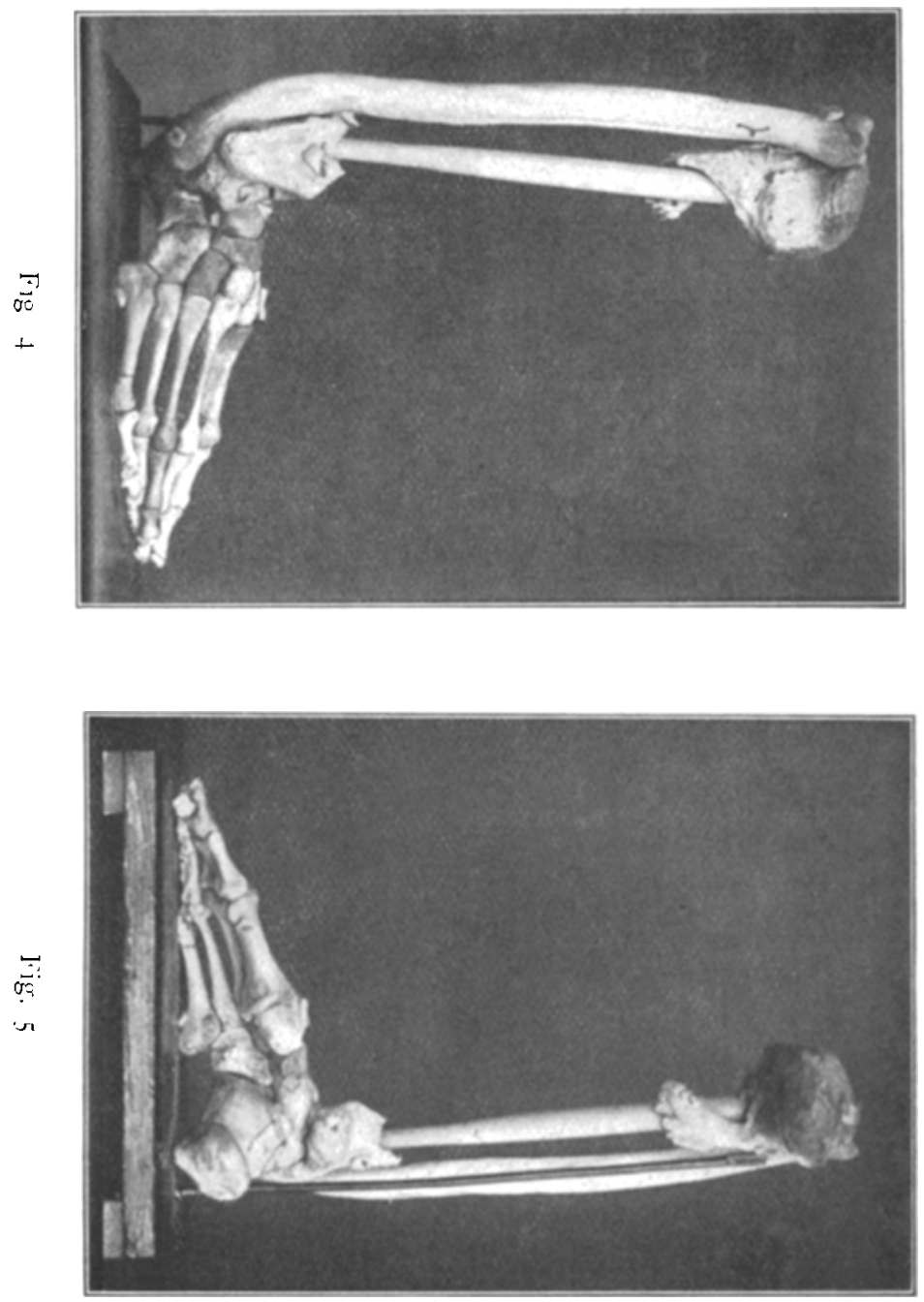

fchlerhafte Stcllung des Fußes, die Klumpfußstellung Auch die Knochenspange an der proximal'n Tibia Epiphyse ist gut zu schen.

Wahrend die anatomischen Veranderungen der Fibula sofort bei Betrachtung von Rontgenbild und P'raparat dem Benchaurer in die

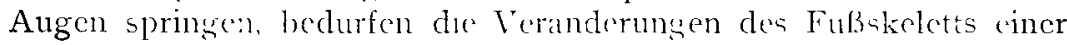


naheren Entersuchung. Ohne weiteres mupuniert der Fub al, Klumpfub, und swar sehore er zu den Arten ron Klumpeuben, dic wir gelegentlich nach schlecht geheilten I nterschenkeltrakturen, hauptnachlich aber nach partellen Renektionen und Nekrotomion der l'sbra bei

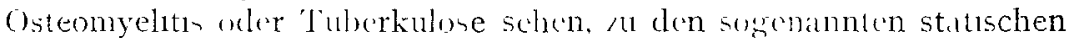
Klunptaben. Samtlehe Verinderungen resultioren atus dem pathologischen Verlatuf der Arhse den aboren Sprungereienk Duse verlicuft normalorweise horimntal, 11 unserem Falle etwa in cinem< ron t5 Grad ron medial oben nach lateral unten. I) fe fibulare Gre

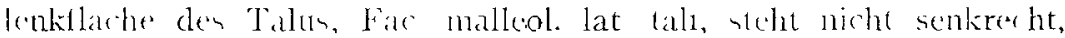

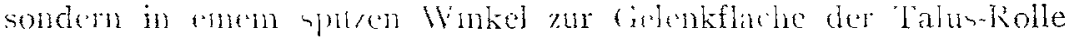

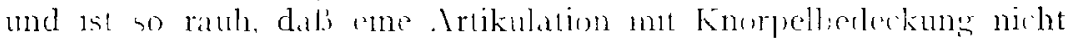
statgetunden haben kann doie methale Grenkflache tur die. Tibia, Iatc malleol. med. tall, die senst in he deulleh ron der Truchlat ab-

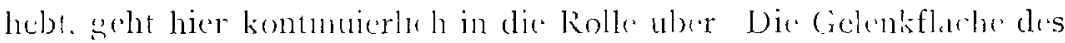

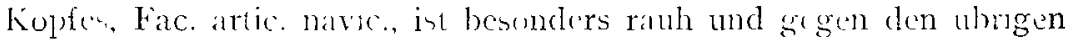
Knochen als ciclenklache nirht absenzbar. Em Prom tromblearis und der Rinne fur alen Nune peron long tehlen vollkommen fine

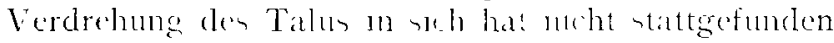

Beinn (alcaneus int folgriden zu benerken: 1) le 3 nomalerweise mit dem 'Talus artikulierenden Cichenkflachen des calcaneus sind

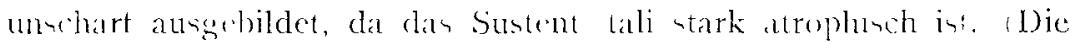

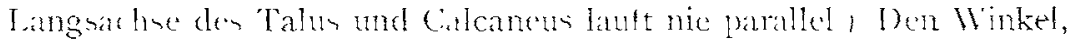

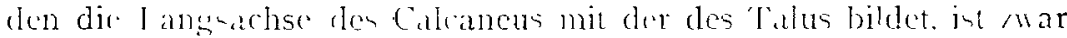
immer noch con stumpter aber wenentlieh spitar als nomml Der Calcaneus steht in feder Richtung des Ralumes utriler fum T.ılus als

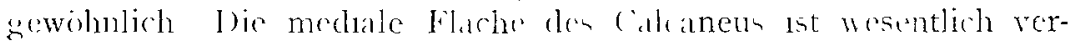
kuret, whorent: die laterate normal geblinben ist, infolgedessen int dic

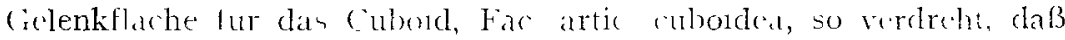
ihr medlaler kand nach hinten geruekt ist.

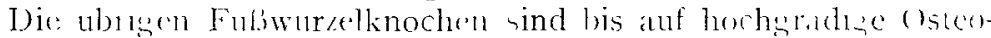
porose nicht verendert. Netatarsue und l'halangen sind ohnt pather-

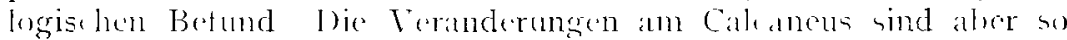
atark, dab durch sio allein ene Ammiherung der Tuberositan usis

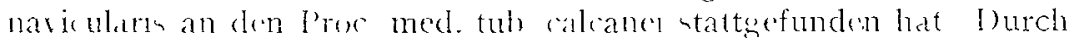

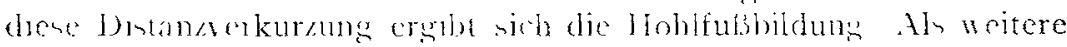
Folge stehen nun auch die Mitelfubknochen mit den Zehen in wiom

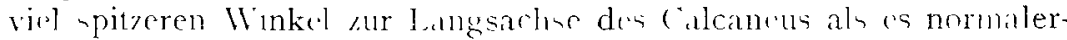
weine der Fall ist

Ziehen wr aus dieser Deformierung des L'nterschenkels und FuBes unsere Rückschlusse auf die Entstehung derselben, so ergibt sich, dal die feste Verankerung der Magnalium-Prothese mit clen Tibiaepiphysen das Längswarhstum des Unterschenkels gestort hat. Die Fibula konnte nicht in der Längsrnchtung weiter 
wachsen, sondern legte sich bogenförmig um die Tibia herum. Hierdurch wurde auch der Fuß aus seiner richtigen Lage gebracht, die Achse des Sprunggelenks konnte nicht mehr horizontal verlaufen. Durch die Belastung resultierte dann eine weitere Verkrümmung der wachsenden Fibula und eine ausgesprochene Klumpfußstellung.

Ein anderes Resultat wäre wahrscheinlich erreicht worden, wenn zur Implantation nicht totes, sondern lebendes Material verwendet worden wäre. So konnte z. B. die Tibia durch die Fibula ersetzt werden, wie ja überhaupt an zwei- und mehrknochigen Gliedern ein Knochen zum Ersatz des Nachbarknochens herangezogen werden kann. Die Entwicklung der modernen Knochenchirurgie ist besonders in den letzten Jahren so ungemein gefördert worden Plastik mit gestielten Knochenlappen, freie Knochentransplantation - , daß man mit Implantation von Fremdkorpern als Knochenersatz besonders bei jugendlichen Individuen äußerste Zurückhaltuns wahren sollte.

\section{Literaturübersicht.}

I. Chratot d d s, Behandlung großerer Knochendefekte. Inau.-Diss. igó, Berlin.

2. Eulenburg Realencyclop. 1913, IV. Aufl.

3 Gluck. Zeatschr, f. arztl. Fortbildg. 1917, Nr. 23 u. 24, 1918, Nr. 1

t - Arch. f klm Chrr. I8yo, Bd. 4 .

5. - Deutsche med. Wochenschr. 1888.

6 - Berlmer klm. Wochenschr. 1899

7 Kamptz Beitr. z. kin. Chir. 1914

8. I. a 11 ge. Jahreskurse f. arztl. Fortbildg. 1920, Dez.-l Ieft

9 Pay r. Arch. f. klin. Chir. 1903, Bd LXXII, H. I

Io - -.. Deutsche Ges. f. Chrr. 1900, XXIX. Kongr.

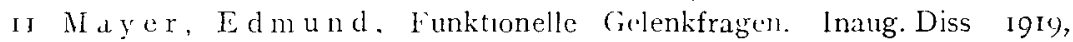
IBerlın. 